\title{
Corela
}

Cognition, représentation, langage

HS-32 | 2020

Les postures énonciatives

\section{De la posture énonciative à la diathèse : gestion et gestation du sens}

\section{Marion Colas-Blaise}

\section{(2) OpenEdition}

\section{Journals}

Édition électronique

URL : https://journals.openedition.org/corela/12082

DOI : 10.4000/corela.12082

ISSN : 1638-573X

Éditeur

Cercle linguistique du Centre et de I'Ouest - CerLICO

Référence électronique

Marion Colas-Blaise, « De la posture énonciative à la diathèse : gestion et gestation du sens », Corela [En ligne], HS-32 | 2020, mis en ligne le 05 novembre 2020, consulté le 14 juillet 2021. URL : http:// journals.openedition.org/corela/12082 ; DOI : https://doi.org/10.4000/corela.12082

Ce document a été généré automatiquement le 14 juillet 2021.

\section{(c) (i) (2) (2)}

Corela - cognition, représentation, langage est mis à disposition selon les termes de la licence Creative Commons Attribution - Pas d'Utilisation Commerciale - Partage dans les Mêmes Conditions 4.0 International. 


\title{
De la posture énonciative à la diathèse : gestion et gestation $\mathrm{du}$ sens
}

\author{
Marion Colas-Blaise
}

1 Pour commencer, essayons de circonscrire un champ de questionnement. En quoi la notion de posture énonciative (stratégies de sur-, sous- et co-énonciation) (entre autres Rabatel 2007, 2008a, b, 2012a, b), permet-elle de rendre compte de la manière dont des instances, locutrices et/ou énonciatives ${ }^{1}$, prennent position dans le monde et rendent ce dernier signifiant? En quoi conduit-elle à étudier les modalités de l'orchestration des sensations, perceptions, opinions et jugements qui entrent dans la construction d'un univers de sens, notamment fictionnel? Quelle en est la valeur opératoire, qui peut être vérifiée à travers des études de cas? Enfin, dans quelle mesure y a-t-il des zones frontières, de flou, où la distinction entre les trois postures résiste à l'analyse et constitue peut-être, en dernier ressort, une question d'interprétation plus ou moins contextualisée? L'identification des postures est loin d'être mécanique: elles continuent à nous interpeller, et c'est en cela que réside tout leur intérêt. $\mathrm{Si}$, dans ses travaux, Alain Rabatel confère aux contours notionnels une grande acuité, il note aussi, dans l'article intitulé "Les enjeux des postures énonciatives et de leur utilisation en didactique » (2007) que la différence entre la sur- et la sous-énonciation peut être ténue, cela en raison d'une « interaction dialogale dissensuelle inégale».

2 Mon hypothèse de départ peut alors être formulée en ces termes : certains cas-limites, particulièrement fréquents dans Un balcon en forêt de Julien Gracq - un roman qu'Alain Rabatel a lui-même sollicité dans Homo narrans (2008a) -, appellent une analyse en termes de co-énonciation (hétéro- ou auto-dialogique), au-delà ou en deçà des distinctions tranchées entre co-, sur- et sous-énonciation. Une condition s'impose d'emblée: il faut prévoir un espace de la co-énonciation, traversé par différentes logiques et sous-tendu par différents régimes de la co-énonciation.

3 Étudiant des exemples empruntés au Balcon en forêt, je viserai, ainsi, à mettre à nu les forces (centripètes ou centrifuges, consensuelles ou dissensuelles) qui sous-tendent la 
co-énonciation et permettent de la distinguer de la sur- et de la sous-énonciation. Pour cela, j'approcherai la notion de posture énonciative à nouveaux frais, en dégageant une structure actantielle, un socle narratif sous-tendant la perception, mais aussi l'attribution du rôle énonciatif à une ou plusieurs instances dans un univers de sens donné. Cela à deux conditions : à condition que le modèle narratif ainsi convoqué comporte une base sensible, c'est-à-dire qu'il trouve son ancrage dans une narrativité « inchoative » (Ricoeur 1983, 1984, 1985) ${ }^{2}$ mettant en forme l'expérience elle-même ; enfin, à condition qu'il intègre des logiques tensives graduelles. C'est sur des bases raffermies que l'attention sera ensuite focalisée sur l'espace de la co-énonciation.

Plus précisément, je relèverai le défi de montrer en quoi la notion de diathèse, héritée de Tesnière (1959), revue par Claude Muller (2006) et Franck Neveu (2004) et réinterrogée, en sémiotique, par Jean-François Bordron (2012), permet de préciser les forces agissant à la base de la co-énonciation. Il s'agira d'explorer l'arrière-plan sémiolinguistique de la posture de la co-énonciation par rapport aux postures de sur- et de sous-énonciation en dégageant la structure actantielle sous-tendant la perception et, plus largement, la distribution des instances énonciatives dans le champ du discours (première partie), avant de postuler une proto-posture énonciative qui est caractérisée par une sensibilité générale (troisième partie). Il importera de montrer en quoi des constructions nominales, des groupes adjectivaux et des participes adjectivés déplacent le regard vers une logique "présentative ", plutôt que représentative (Combe 1991: 149), c'est-à-dire pré-réflexive (Guillaume 1964, 1965 [1929]). Là où, justement, l'indifférencié l'emporte sur la différenciation des postures. L'hypothèse à vérifier est qu'ils témoignent au niveau du texte de l'émergence du pathique, d'une instance "impersonnelle", en deçà ou au-delà de l'actantialité et de la narrativisation proprement dites. Quant à la deuxième partie, elle visera à mettre à nu les logiques tensives à la base de l'interaction des instances énonciatives. Plus précisément, je remettrai sur le métier la conception de la co-, sur- et sous-énonciation en termes de concordance et de discordance (Rabatel 2007).

5 Mon cadre théorique se construira ainsi au carrefour de la théorie du point de vue développée par Alain Rabatel, des Eléments de syntaxe structurale de Tesnière, de la théorie psychomécanique du langage et de la sémiotique (sémiotique de la perception (Bordron 2011), sémiotique tensive (Zilberberg 2012)).

\section{Les postures énonciatives et la diathèse actantielle : une première caractérisation de la co- énonciation}

6 Je définis la diathèse comme l'« attitude de l'énonciateur par rapport à la façon dont il exprime l'énoncé » ${ }^{3}$ (Bordron 2012), comptant, par ce biais, revisiter les notions de position, positionnement et posture chères à Alain Rabatel (2012a). Plus linguistiquement, la diathèse est considérée comme la description d'une configuration verbale régissant les relations entre des rôles sémantiques et des fonctions syntaxiques. D'une part, elle permet de conférer une structure actantielle à la perception, qui est, avec la pensée, au départ des $\mathrm{PDV}^{4}$ et des postures énonciatives; d'autre part, elle invite à rendre compte des stratégies médiationnelles à la base de l'installation des postures énonciatives dans un univers de sens. 
7 Ainsi, l'on postulera une définition interactionnelle de la perception compatible avec la manière dont Alain Rabatel conçoit les relations entre une instance énonciative et la réalité. En articulant la diathèse active avec la diathèse passive (le sujet n'est pas seulement agissant, mais agi) et avec la diathèse réciproque (simultanéité des procès actif et passif; Tesnière 1959: 34), voire avec la "diathèse réciproque fusionnée " (Bordron 2012 : «Alfred et Bernard s'entretuent », « un seul amour partagé »...), il est possible de décrire l'interaction entre l'instance percevante et la réalité comme une cofondation. La réalité signifie pour moi à travers la perception et je suis «moi » en me situant par rapport à la réalité en tant que percepteur. La perception peut alors être considérée comme une « activité » (Bordron 2012).

8 En ce qui concerne les postures énonciatives, il est nécessaire de remonter vers l'activité scripturale elle-même et de montrer en quoi la notion de diathèse peut rendre compte de la gestion des PDV, c'est-à-dire de la distribution des délégations et de la multiplication ou de la diminution du nombre des médiations énonciatives ${ }^{5}$. Nous dirons que la diathèse causative selon Tesnière, c'est-à-dire le «faire faire ", qui a pour effet l'accroissement du nombre des intermédiaires et la création d'une distance (débrayage), engage une visée centrifuge, alors que la diathèse récessive, la suppression des intermédiaires, est liée à l'embrayage et à une visée centripète. Les deux diathèses constituent ainsi des pré-formes qui gèrent l'orchestration des instances dans leur rapport à l'événement de sens.

9 Considérons les PDV dans un premier extrait d'Un balcon en forêt :

(1) Dès qu'on avait dépassé les dernières maisons de Moriarmé, le goudron cessait, tandis qu'on entamait les premiers lacets. On eût dit que la caillasse de la route avait été charruée sur toute sa largeur : c'était une sorte de reg saharien, un fleuve de pierres sans fossé ni banquette entre les deux murs des taillis. Grande consulta sa carte parmi les cahots : on s'engageait dans une laie forestière (p. 16) ${ }^{6}$.

10 Attardons-nous sur la deuxième phrase. Le pronom indéfini « on » (« on eût dit que la caillasse de la route... ») traduit une co-énonciation, non seulement entre énonciateurs seconds unis dans une même perception/pensée, mais encore entre le Locuteur/ Énonciateur premier et un ou plusieurs énonciateurs seconds (accord par validation et justification communes, ici implicites). Le narrateur peut se mettre empathiquement à la place du ou des personnages, engagé(s) dans un monologue intérieur infra-verbalisé. Dans ce cas, nulle discussion, nulle négociation des positions, mais, du point de vue actantiel, une belle coïncidence ou accord : le « on " possède une "valeur de marqueur d'un récessif intégral » (Bordron 2012). Je le mets en relation avec l'opération de l'embrayage et une visée centripète. On assiste à un télescopage, voire à une fusion des instances énonciatives. Enfin, selon une suggestion d'Alain Rabatel ${ }^{7}$, à ce premier type de co-énonciation s'en ajoute un autre, auto-dialogique, si l'on admet que la coénonciation, par opposition à la sur- et à la sous-énonciation, est fondée sur l'adéquation entre la perception et l'interprétation.

11 En même temps, la distance avec l'événement demeure, comme en témoigne le subjonctif plus-que-parfait à valeur de conditionnel passé : l'identification de l'événement est proposée à titre d'hypothèse (irréel du passé), c'est-à-dire préassertée par le Locuteur/Énonciateur premier et préassumée par l'énonciateur second. Je réserve ici la gamme des assertions - assertion, désassertion, prise en compte, préassertion (cf. Colas-Blaise 2011) - au locuteur/énonciateur et la gamme des assomptions - assomption, désassomption, non assomption, préassomption - aux 
instances responsables d'un PDV qui ne sont pas locutrices. Selon Jacques Fontanille (2003 [1998] : 282-283), la "prédication assomptive" permet à une instance de faire connaître sa " position par rapport à ce qui advient dans son champ ». Ou encore, «la prédication assomptive est une autre articulation de la présence, complémentaire de la première [la prédication existentielle grâce à l'assertion]. D'une certaine manière, il s'agit en effet de la présence à l'autre, présence de l'instance de discours à ce qui advient, présence à ce qui apparaît dans le champ et qui n'est pas elle-même ». Il s'agit ici d'adapter la notion d'assomption, qui peut être découplée de celle d'assertion, à un cadre hétéro- ou auto-dialogique.

La valeur modale (« on eût dit ») peut être du ressort du Locuteur-Énonciateur premier, qui co-énonce. Se pourrait-il aussi - lecture concurrente - qu'il prenne ses distances par rapport au PDV du personnage sans pour autant adopter la posture du surénonciateur? L'analyse est délicate, en raison de l'absence des marques de reprise, citation, mention ou reformulation qui entrent souvent dans la co-construction du PDV (Rabatel 2012a). Dans le cas du compte rendu en style indirect libre, il est possible que le Locuteur/Énonciateur premier se fasse l'écho du PDV du personnage, sans marque de distanciation. Il asserte, non point le PDV de l'énonciateur second, mais le fait que ce dernier a interprété le paysage. Il se contente alors d'enregistrer le PDV de l'énonciateur second, qui propose une interprétation de l'événement de sens. Il prend acte du PDV de l'énonciateur second ${ }^{8}$. Nous sommes ainsi confrontés à une indétermination du sens, que le contexte permet toutefois de réduire.

On en conclura, prudemment, que si l'hétéro- et l'auto-dialogisme constituent deux types de co-énonciation, cette dernière s'exprime aussi en intensité et en étendue. L'expression "on eût dit " suggère la possibilité de distinguer une co-énonciation tonique (première interprétation) et une co-énonciation atone (deuxième interprétation). Enfin, la portée de l'assomption varie dans les deux cas.

C'est ce que nous chercherons à vérifier en considérant la suite de l'extrait. Qu'implique le double-points introduisant le style indirect libre ? Ce dernier est associé à la montée vers l'assertion/assomption "c'était une sorte de reg saharien... ». "On » dit et/ou pense qu'il est vrai que la caillasse est "une sorte de reg saharien». L'expression " une sorte de » s'insère dans une chaîne de modalisations qui sont autant de traces de l'inscription d'une subjectivité (inadéquation possible de la dénomination ; « une sorte de » ne traduit ni un acte d'identification ni un acte de rupture, mais un acte de différenciation (Rabatel 2012a)). On peut parler d'une co-énonciation (mise au même niveau du Locuteur/Enonciateur premier et de l'énonciateur second) - même si elle reste implicite -, d'une « récession » du point de vue de la diathèse et d'une visée centripète (embrayage). La co-énonciation porte sur le cadre de l'assertion/assomption et/ou sur la dénomination, voire sur le fait de mettre en doute la justesse de la dénomination. Dans ce dernier cas, nous parlons d'une co-énonciation tonique (même si elle reste implicite) parce que l'investissement et la force d'engagement sont facilités, d'une part, par l'emploi du terme technique « reg ", qui témoigne d'un savoir commun aux énonciateurs et validé comme tel ${ }^{9}$, et, d'autre part, par un accord interne - voire intime - sur l'inadéquation possible de la dénomination. $\mathrm{Si}$, toutefois, " une sorte de " témoigne des réticences d'un des énonciateurs par rapport au PDV d'un autre et que la mise en doute de l'adéquation du terme «reg» ne soit donc pas partagée, la coénonciation est dite atone. 


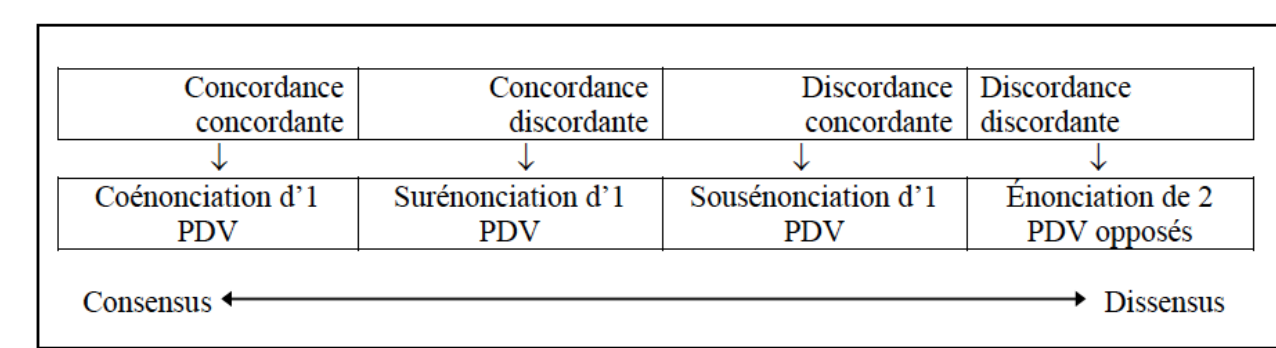

Dans ce contexte, la scription italique peut non seulement indiquer la présence d'un terme technique ou étranger, mais encore traduire une "énergétique du mot ", selon les termes de Julien Gracq (1977), c'est-à-dire un pic d'intensité et un investissement maximal de l'instance énonciative (aux niveaux 1 et/ou 2) qui s'approprie ce mot emprunté à un langage technique.

Résumons. La co-énonciation peut être approchée sous différents angles :

(i) du point de vue de la diathèse mise en œuvre. La notion de diathèse permet alors de caractériser la co-énonciation par rapport à la sur- et la sous-énonciation. Si, dans le cas de la sur- et de la sous-énonciation, la diathèse causative de l'ordre du "faire faire » serait rapprochée de l'opération du débrayage (déhiscence et mise à distance) et d'une visée centrifuge, dans le cas de la co-énonciation, la diathèse est récessive; elle correspond à un embrayage (consubstantialité des énonciateurs) et à une visée centripète. Ainsi, les postures énonciatives se définissent au croisement de différents régimes de la prise en charge/assertion et assomption et de constellations énonciatives dont la taille varie en fonction des médiations et des délégations mises en oeuvre (diathèse récessive ou causative; visée centripète ou centrifuge; embrayage ou débrayage) ;

(ii) du point de vue des types de co-énonciation. On peut distinguer deux types de coénonciation : hétéro- et auto-dialogique ;

(iii) du point de vue des variations en intensité et en étendue. La co-énonciation peut s'exprimer en intensité - variantes tonique et atone - et en étendue (portée).

Par définition, la co-énonciation atone n'est pas exempte de tensions. Dans la deuxième partie, il s'agira de préciser davantage celles qui peuvent traverser son espace. À terme, nous comptons requalifier les variations en intensité et en étendue en régimes de la coénonciation.

\section{Concordance/discordance et logiques tensives}

1 Nous adossons notre réflexion au schéma qui permet à Alain Rabatel et Solveig Lepoire Duc (2005) d'envisager les relations entre les énonciateurs :

Alain Rabatel, Solveig Lepoire Duc (2005)

Ce schéma jette les bases d'une lecture attentive aux tensions qui se nouent ou se dénouent le long de différentes lignes tensionnelles. Mon hypothèse est que la concordance concordante et la discordance discordante mettent en œuvre une logique « implicative », de l'ordre du si x... alors $y$, la concordance discordante et la discordance concordante donnant lieu à une logique « concessive » $x$ bien que $y$ (Zilberberg 2011) ${ }^{10}$. D'un côté, le parvenir (logique implicative) se solde par un consensus ou un dissensus, donc par une forme d'équilibre (co-énonciation ou juxtaposition de PDV incompatibles) 
; de l'autre, le survenir (logique concessive) caractérise le retournement de situation, le surgissement de l'événement, un moment d'intensification et une forme de déséquilibre, comme moteur de la narrativité (sur- et sous-énonciation).

La réalité des textes nous demande, cependant, de complexifier ce schéma. Interprétant librement une suggestion d'Alain Rabatel ${ }^{11}$, nous essayons de vérifier l'hypothèse que la logique implicative comprend elle-même des moments de blocage et de relâchement de la tension, tout comme la logique concessive comporte des moments où la tension s'accroît sans heurts ${ }^{12}$. Ainsi, la co-énonciation, c'est-à-dire la construction d'un PDV commun, peut intégrer une part de dissensus. Je distinguerai trois cas: i) les coénonciateurs rejettent conjointement un PDV tiers jugé antagonique: ii) l'accord (explicite ou implicite) tolère - du moins provisoirement - une dose de dissensus entre eux; iii) les co-énonciateurs se mettent d'accord sur le fait qu'il y a une dose de dissensus. La co-énonciation dissensuelle consiste dans ce cas moins à éclairer différemment un objet de sens commun qu'à co-construire, éventuellement dans le conflit, deux objets de sens différents ${ }^{13}$.

En ce sens, la co-énonciation n'est pas exempte de moments conflictuels où la tension se noue. L'accord entre les co-énonciateurs implique des négociations ${ }^{14}$ qui peuvent déboucher, à terme, sur un désaccord. Nous sommes ainsi en mesure de croiser les variantes tonique et atone de la co-énonciation avec deux régimes de la co-énonciation distincts: consensuel et dissensuel. Il paraît avantageux de parler d'un espace de confrontations placé, globalement, sous le signe de l'accord ${ }^{15}$, où se logent une coénonciation consensuelle, tonique ou atone, ainsi qu'une co-énonciation dissensuelle, tonique ou atone.

Symétriquement, on peut avancer que le désaccord sous-jacent à la coexistence de PDV antinomiques n'exclut pas des moments ponctuels de pacification et de mise en commun. Il peut accepter une dose de consensus, avant de déboucher, éventuellement, sur un (nouvel) accord conquis sur le désaccord ${ }^{16}$. La concordance concordante consensuelle et dissensuelle et la discordance discordante dissensuelle et consensuelle doivent être distinguées de la concordance discordante et de la discordance concordante selon Rabatel: en effet, elles se placent sous les régimes de la coénonciation et de l'énonciation de deux PDV opposés et non sous les régimes de la suret de la sous-énonciation ${ }^{17}$.

Résumons : 


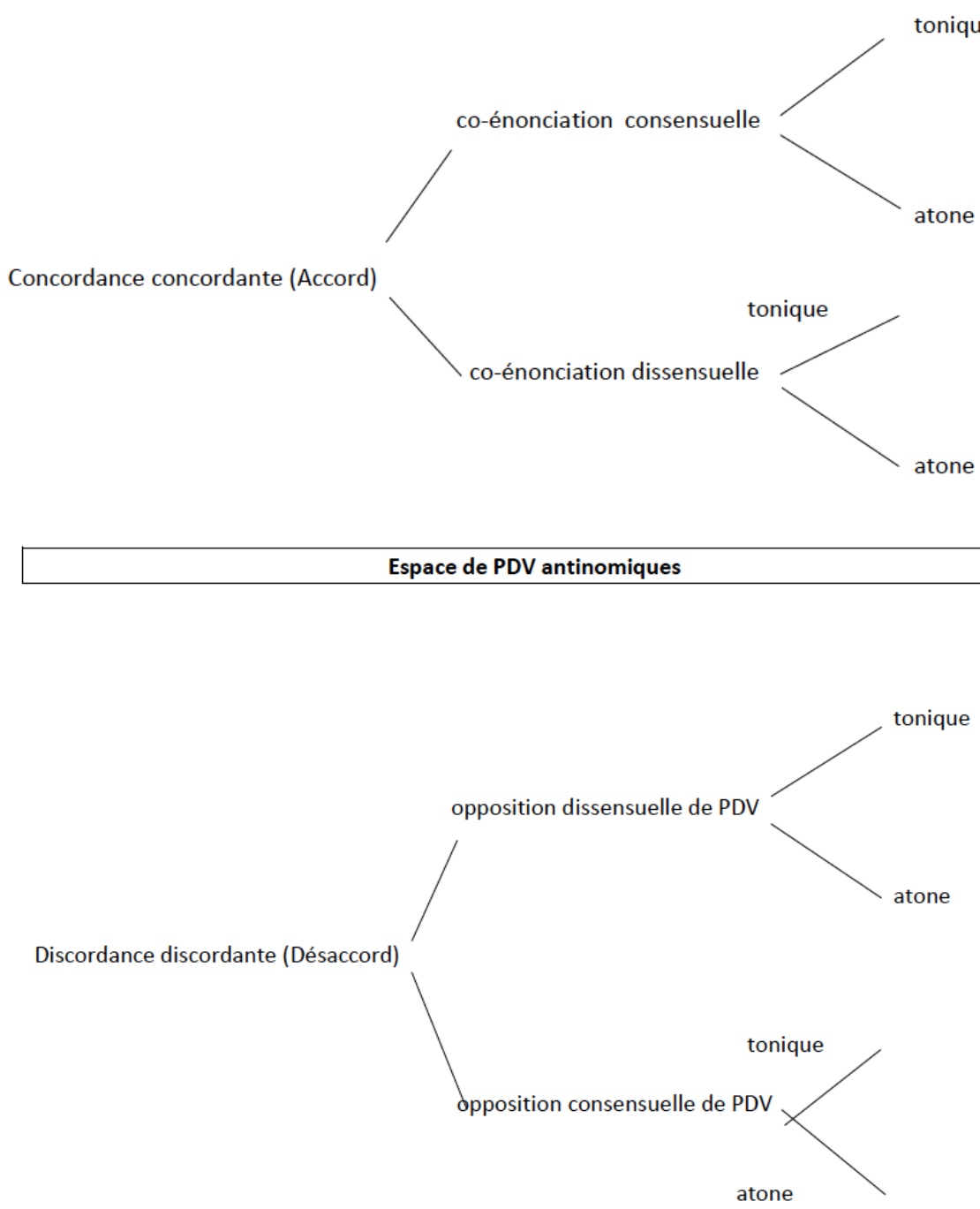

Dans Un balcon en forêt, l'accord dissensuel (tonique ou atone) semble céder la place, très souvent, à l'accord consensuel atone (construit sur le décalage entre les PDV des co-énonciateurs plus que sur un véritable conflit). L'exemple (2) admet deux interprétations :

(2) La solitude était complète, et cependant l'idée d'une rencontre possible ne disparaissait pas complètement: quelquefois on croyait distinguer dans l'éloignement un homme debout au bord de la chaussée sous sa longue pèlerine : de près, c'était un petit sapin tout noir et carré d'épaules contre le rideau de feuilles claires (pp. 19).

À propos du début de l'extrait, l'on parlera de compte rendu de perceptions/pensées au style narrativisé (ici au second plan), avant que le contenu de la perception ne soit explicité sous la forme d'un compte rendu au style indirect libre : « un homme debout au bord de la chaussée sous sa longue pèlerine » et, surtout, « de près, c'était un petit sapin tout noir et carré d'épaules contre le rideau de feuilles claires ». On y verra, une nouvelle fois, un PDV co-construit, le style indirect libre relevant, ici, d'une logique implicative. Les points de vue du Locuteur/Énonciateur premier et de l'énonciateur 
second se mêlent et tendent vers une concordance concordante. Le développement du PDV co-construit concerne l'axe tensif de l'étendue: de la même manière que le déroulement de la phrase occupe l'espace et le temps, le regard consomme du temps en enjambant le ici et le tout près en direction du "là ", correspondant à la zone proximale, et du « là-bas ", caractéristique de la zone distale (Rastier 2002 : 248-249). La rupture locale s'accompagne d'une rupture modale, qui oppose le certain (en relation avec le je-ici-maintenant) au probable (là) et à l'irréel (là-bas). Ce franchissement d'un seuil est confirmé, dans (2), par une relative incertitude, dont témoigne le verbe " croire » (« on croyait distinguer »).

En même temps, en vertu d'une interprétation alternative, on peut déceler une coénonciation faiblement consensuelle, c'est-à-dire atone, en jouant sur la dissimilation, au sein même de l'accord manifesté par "on", entre l'Énonciateur qui "sait » et l'énonciateur qui «croit». Dans le contexte général, l'exemple atteste moins une surénonciation qu'un décalage entre les PDV co-construits. Le style indirect libre peut en effet correspondre à une échappée permettant au PDV de Grange de se déployer, même si celle-ci est immédiatement médiée par le PDV du L/E. En témoignent les marques de subjectivité à mettre à son compte (surtout l'adverbe d'intensité «tout » modifiant le qualificatif « noir » et l'inscription sur l'isotopie « humain » (« carré d'épaules»)). Les deux points agissent à l'instar du double tiret qui, dans l'exemple (3), met en œuvre une logique rythmée par la rupture et la reprise et encadre la description aspectualisée du milieu environnant :

(3) La forêt était courtaude - c'étaient des bouleaux, des hêtres nains, des frênes, de petits chênes surtout, ramus et tordus comme des poiriers - mais elle paraissait extraordinairement vivace et racinée, sans une déchirure, sans une clairière; $(\mathrm{p}$. 19).

L'esprit de Grange s'échappe vagabond, exemplifiant ce qu'à la suite de Michel Murat, on peut appeler le braconnage d'esprit.

Dans l'exemple (4), le compte rendu au style direct autonomise davantage le PDV du personnage et peut conforter l'idée d'une confrontation de PDV distincts :

(4) Où me mène-t-on? songeait-il. Il calcula que depuis la Meuse on avait dû faire une bonne douzaine de kilomètres : la Belgique ne pouvait être loin (p. 20).

Une nouvelle fois, après le compte rendu au style indirect à mettre au compte du narrateur, l'on ne constate aucune forme de dissensus (implicite ou explicite) ni de contestation qui corroborerait une vraie déhiscence entre deux niveaux de manifestation du PDV. L'intensification subjective cumulant dans le style direct se monnaye toutefois dans la suite du texte (« une bonne douzaine de kilomètres », «la Belgique ne pouvait être loin »).

Que faut-il retenir de ces explorations ? D'une part, élargissant la perspective, l'on peut concevoir un continuum borné par la concordance concordante et la discordance discordante (les sur-contraires selon Zilberberg encadrant les sous-contraires concordance discordante et discordance concordante) et une version forte et une version faible de la co-énonciation consensuelle ou dissensuelle (cf. Rabatel 2012a pour une approche scalaire et des hésitations entre interprétations) ${ }^{18}$. D'autre part, dans Un balcon en forêt, la concurrence entre le PDV du Locuteur/Énonciateur premier et celui de l'énonciateur second (Grange) est possible, quoique très affaiblie. Pour rendre compte de l'espace des confrontations, on hésitera, selon les cas de figure, entre une 
co-énonciation consensuelle atone et, éventuellement, une co-énonciation dissensuelle atone.

De fait, plutôt que d'un vrai dissensus, les extraits invitent à rendre compte de l'étape inchoative d'un décalage qui, du point de vue topologique, consiste à creuser un intervalle, l'écart, au sens où l'entend François Jullien (2012) ${ }^{19}$. L'établissement de la différence, note ce dernier, suppose une vue en surplomb et une position d'extériorité ${ }^{20}$, ce que corrobore la distinction entre PDV dominants et dominés. Tout au contraire, à condition d'être aménagée, la notion d'empathie ${ }^{21}$, à laquelle Alain Rabatel a consacré de nombreux articles (cf. par exemple 2013, 2014), permet, ici, de saisir cet écart fragile : dans le cas de l'empathie, l'émotion est " quasi prise en charge " (nous dirions assumée) par l'énonciateur second, plutôt que d'être exprimée directement par le Locuteur/Énonciateur premier. On peut y voir un mouvement vers, mais aussi une mise en tension et une séparation minimale, par quoi l'écart (plutôt que la différence) peut s'établir ${ }^{22}$. C'est bien ce dont la notion de co-énonciation atone consonante peut rendre compte.

Essayons d'en tirer toutes les conséquences. Les postures énonciatives, telles qu'elles ont été définies, seraient opératoires surtout pour des textes à forte charge actantielle narrative (avec une pluralité d'instances pourvues de rôles, des moments de nouement/dénouement, des revirements, etc.). Dira-t-on que, dans Un balcon en forêt, l'entrecroisement des versions consensuelle et dissensuelle de la posture de la coénonciation, sans que les frontières apparaissent explicitement, du fait qu'il reste en deçà de la production de différences, est emblématique d'une poétique très particulière: d'une poétique du battement ou de l'intervalle, où l'écart retarde l'apparition de la différence et, a fortiori, de la discordance? J'aimerais défendre l'idée que si la distinction des postures énonciatives est basée sur la différence, l'emmêlement des versions consensuelle et dissensuelle de la co-énonciation réclame la prise en compte de l'écart. À cela s'ajoute que le glissement d'une version à une autre, sans que les marques soient toujours très explicites, c'est-à-dire la graduation entre différentes manifestations, tonique et atone, reproduit au plan de l'expression un double mouvement sur le plan de la fiction : d'une part, l'activité du personnage déserteur, qui cherche à creuser un espace-temps à lui, en déport de l'Histoire, de la guerre, et qui, à cette fin, se livre à une activité de perception minutieuse, scrutant, inventoriant le paysage, mieux, y traçant des lignes de différenciation fines. D'autre part, le retrait de Grange sujet sensible et percevant, traduit, notamment, par le pronom «on»: l'estompage des frontières, un flou généralisé anticipent sur la fin, quand Grange blessé finit par s'abandonner à une grande in-différence. La logique narrative rencontre ses limites : l'introjection l'emporte sur la projection et la rétrojection. On peut parler d'une proto-narrativité, où la durée échappe à une temporalisation à travers une articulation en passé, présent, futur. Désormais, et il s'agit de la quasi-fin du livre : « Il n'arriv[e] rien ». Nous avons vu que l'événement (historique) est ramené à l'événement perceptif ; ce dernier finit lui-même par se dissoudre ${ }^{23}$.

Dans la troisième partie, je me demanderai en quoi le rapprochement entre la coénonciation et l'empathie ainsi que la pensée de l'écart nous guident vers une protonarrativité qui sous-tend une proto-posture énonciative. La question paraît importante dès lors que cette remontée vers des couches de sensibilité plus profondes, que l'on mettra en relation avec ce qu'Alain Rabatel appelle le pré-réflexif $\left(2003^{24}, 2008 \mathrm{a}\right)$, n'est pas d'ordre purement spéculatif, mais prend appui sur des marques dans le texte. Il 
s'agira de montrer que des sélections lexicales et des constructions morphosyntaxiques donnent accès à cet amont où des formes émergent à partir de ce que JeanFrançois Bordron (2012), dans sa réflexion sur la diathèse, appelle des formants ${ }^{25}$.

\section{Proto-posture énonciative et « diathèse à formant »}

Notre objectif est donc de remonter vers une proto-posture énonciative, sous-tendant la posture de la co-énonciation étudiée par Alain Rabatel. Cette dernière peut en effet être considérée comme le résultat d'une genèse. Cette idée sera argumentée à partir des travaux de Jean-François Bordron et de Gustave Guillaume.

Jean-François Bordron (2012) appelle "diathèse à formant » des «changements dans l'ordre de la sensibilité » qu'il s'agit non pas de dire, mais d'exprimer. La manifestation linguistique peut exprimer un "état indéterminé ", "une source d'énergie diffuse ", une logique des flux, des forces qui constitue une « logique de l'être » (et non plus de la possession ou de la privation, comme pour le modèle narratif).

Ensuite, retrouvant la notion de tension dans un cadre théorique différent de celui de la sémiotique, je convoque également la distinction guillaumienne entre la première chronothèse in posse, la deuxième chronothèse in fieri et la troisième chronothèse in esse, pour opposer l'être devant le temps (virtuel), l'être au temps (émergent) et l'être dans le temps (achevé), à la base du narratif. Adoptant le point de vue de la genèse de la perception et de la posture énonciative, je vise à retrouver la temporalité « originaire » de la première chronothèse qui est encore impersonnelle. L' "état d'âme", selon la " diathèse à formant », est encore diffus et caractérise une strate d'organisation du sens avant toute individuation et toute dissociation des énonciateurs. La proto-posture émerge à la croisée entre flux et reflux, entre attractions et répulsions. On peut distinguer de l'empathie qui, nous l'avons vu, repose sur la proximité, voire sur la connivence des énonciateurs, et de la sympathie qui, selon Alain Rabatel (2014), consiste en une identification aux autres, le pathique, au ras du sentir, des sensations encore mêlées.

D'abord, on constate que, dans Un balcon en forêt, le régime de la co-énonciation peut prendre les dehors du présent générique, élargi, in- ou atemporel ${ }^{26}$ :

(5) À chaque virage en épingle à cheveux, la vallée se creusait, une coulée de brouillard au long de sa rivière qui s'asséchait et glissait vers l'aval, de plus en plus vite, soulevée de remous, comme l'eau d'un bain qui se vide. [...] où qu'il fût, comme les enfants qui grimpent aux portières, tout point de vue le magnétisait jusqu'à l'impolitesse (pp. 16-17).

41 L'emploi générique donne souvent lieu à la désactualisation d'un verbe causatif (Larjavaara 2000). Le verbe au présent générique déborde le référentiel énonciatif, au profit du référentiel des savoirs partagés et des vérités générales. Il n'est sans doute pas anodin que dans les deux exemples (« se vide, "grimpent»), le présent générique soit relégué dans une proposition relative adjective, comprise dans une construction nominale. On constate non pas une abolition du narratif - le verbe conjugué reste présent - mais une sorte de résistance à une mise en forme de type actantiel.

Ce mouvement est accentué, dans l'exemple (6), par deux constructions nominales comprenant des déterminants démonstratifs déictiques ${ }^{27}$, mais aussi l'adjectif qualificatif « têtu » ainsi qu'un participe passé adjectivé et deux adjectifs verbaux : 
(6) Seulement cette ombre têtue, voilée, intimidante, qui flottait vers lui sans le

rejoindre du fond de ses limbes vagues - ce silence étourdissant (p. 252). logique actantielle narrative, sur le mode de la diathèse récessive (co-énonciation) par opposition à celui de la diathèse causative (sur- et sous-énonciation); ensuite, en remontant vers une proto-posture énonciative, sous-tendant la co-énonciation. On a constaté, en effet, qu'Un balcon en forêt nous oblige à entrevoir une préforme diathétique "à formant» (Bordron 2012). La manifestation linguistique (la syntaxe nominale au sens large) donne accès à une strate d'organisation du sens où une «sensibilité générale » (Bordron ibid.) tend vers sa spécification sous la forme de PDV, sans encore l'atteindre tout à fait. Elle témoigne de l'émergence de la posture de la coentière", le participe présent engage un mouvement de détension, que le participe passé, en particulier quand il est adjectivé, achève (dépassement de la chronologie narrative et retour à un temps pré-réflexif). Les noms « ombre » et " silence » semblent échapper à tout devenir, voire nient ce dernier (résistance à la lumière et absence de bruit). Il ne s'agit plus de représenter une " réalité », les objets du discours, sous-tendus par une structure narrative, mais de les présenter comme possibles ${ }^{28}$. troisième topothèse, in esse, en relation avec le « ici » (Détrie, Siblot, Verine 2001 : 331), non point à une subjectivité en soi-même, mais à un geste négociant le passage de l'anté-thétique au thétique (Colas-Blaise 2017), là où se trace un premier écart, où se dessine une différence encore liminaire ${ }^{29}$. D'où l'idée que nous assistons dans ces pages à un processus de rétrogression vers une logique présentative qui est en cours, encore fluctuante. La syntaxe nominale nous rend attentifs à la logique du flux et du reflux, de la tension et de la détension. À la fin d'un Balcon en forêt, la manifestation linguistique laisse affleurer un proto-sujet qui se trouve débordé par les sensations, mû par elles (l'ombre est "intimidante » et le silence "étourdissant»). On lit aussi que "cette pensée même ne se fixait pas : il lui semblait qu'elle remontait malgré lui vers des eaux plus légères » (p. 252). Le proto-sujet n'est pas encore (n'est plus) une instance locutive ou énonciative assertant ou assumant. Il n'est pas un quasi-sujet, qui prédique, ni même ce que Jean-Claude Coquet (2007) appelle un "non-sujet », c'est-à-dire un corps qui perçoit ou parle. À partir d'une instance encore «impersonnelle», selon le terme de Guillaume, l'énergie ou le flux/reflux conduisent à l'émergence d'une forme.

On peut ainsi caractériser la notion de posture par rapport à celles, voisines, de position et de positionnement : alors que l'adoption d'une posture énonciative témoigne d'un mode d'entrée en relation de perceptions/pensées (hétéro- ou auto-dialogisme) médiées par le langage et de leur distribution du point de vue actantiel, la notion de positionnement signifie un proto-embrayage ${ }^{30}$ (un être au monde et au temps); quant à celle de position, elle permet de rendre compte de l'attitude d'une instance percevante qui est, dirons-nous d'un point de vue sémiotique, devant le monde ${ }^{31}$.

$\mathrm{Au}$ terme de ce parcours, il apparaît qu'un Balcon en forêt privilégie l'espace de la coénonciation, l'analyse des extraits réclamant des distinctions fines entre l'hétéro- et l'auto-dialogisme, entre les régimes consensuels (plutôt que dissensuels) tonique et atone. Si l'espace de la co-énonciation est traversé de tensions, celles-ci renvoient à une poétique de l'écart signifiant.

Pour finir, résumons tous les enjeux de cette réflexion: d'abord, en dégageant une 
énonciation proprement dite, là où se décident les tensions qui, ultérieurement, prennent la forme de la consension et de la dissension, où le flux et le reflux préparent l'activité interactionnelle dont la notion de posture énonciative permet de rendre compte. Prendre en considération cette gestation du sens, c'est rétablir en amont de l'empathie, toujours négociée, ainsi que le montre Alain Rabatel dans ses écrits, le pathique, les sensations emmêlées, cette disposition encore diffuse - cet "état énergétique de notre âme » (Bordron ibid.) avant toute individuation - qui la sous-tend.

\section{BIBLIOGRAPHIE}

Baroni, Raphaël, 2010/3, « Ce que l'intrigue ajoute au temps. Une relecture critique de Temps et récit de Paul Ricœur », Poétique, n 163, pp. 361-382.

Bertrand, Denis, 2005, « Deixis et opérations énonciatives », in D. Monticelli, R. Pajusalu, A. Treikelder (éds), « De l'énoncé à l'énonciation et vice versa. Regards multidisciplinaires sur la deixis », Studia Romanica Tartuensia, IVa, Tartu University Press, pp. 171-185.

Bordron, Jean-François, 2011, L’iconicité et ses images. Études sémiotiques, Paris, PUF.

Bordron, Jean-François, 2012, «Vie(s) et diathèses ", Actes sémiotiques, $\mathrm{n}^{\circ} 115$. Disponible sur : <http://epublications.unilim.fr/revues/as/2654> (consulté le 14/07/2018).

Colas-Blaise, Marion, 2011, « Les types et les régimes de la prise en charge », in P. Dendale, D. Coltier (dirs), La prise en charge énonciative. Études théoriques et empiriques, Bruxelles, De Boeck Duculot, pp. 37-54.

Colas-Blaise, Marion, 2012, « Forme de vie et formes de vie », Actes sémiotiques, $\mathrm{n}^{\circ} 115$. Disponible sur : <http://epublications.unilim.fr/revues/as/2631> (consulté le 14/07/2018).

Colas-Blaise, 2017, « Manières d'être et de faire simple. Éléments pour une approche sémiotique de la simplicité », in S. Jollin-Bertocchi, L. Kurts-Wöste, A.-M. Paillet \& C. Stolz, La simplicité. Manifestations et enjeux culturels du simple en art, Paris, Honoré Champion, pp. 357-372.

Combe, Dominique, 1991, La pensée et le style, Paris, Éditions Universitaires.

Coquet, Jean-Claude, 2007, Phusis et logos. Une phénoménologie du langage, Paris, Presses universitaires Paris 8, Saint-Denis.

Détrie, Catherine, Siblot, Paul, Verine, Bertrand, 2001, Termes et concepts pour l'analyse du discours. Une approche praxématique, Paris, Honoré Champion.

Ducrot, Oswald, 1984, Le dire et le dit, Paris, Minuit.

Fontanille, Jacques, 2003 [1998], Sémiotique du discours, Limoges, Pulim.

Gracq Julien, 1958, Un balcon en forêt, Paris, José Corti.

Gracq, Julien, 1977 [1946], André Breton, quelques aspects de l'écrivain, Paris, José Corti.

Groupe $\mu, 2015$, Principia Semiotica. Aux sources $d u$ sens, Bruxelles, Les Impressions Nouvelles.

Guillaume, Gustave, 1964, 1965 [1929], Temps et verbe, Paris, Honoré Champion. 
Jullien, François, 2012, L'écart et l'entre. Ou comment penser l'altérité, FMSH-WP-2012-03.

Larjavaara, Méri, 2000, Présence ou absence de l'objet. Limites du possible en français contemporain, Academia Scientiarum Fennica.

Monte Michèle, 2011, «Intersubjectivité et coénonciation dans la poésie contemporaine », in B. Verine \& C. Détrie (dirs), L'actualisation de l'intersubjectivité : de la langue au discours, Limoges, Lambert-Lucas, pp. 199-138.

Muller, Claude, 2006, « Diathèses et voix en français : une répartition des rôles », in H. Nølke, I. Baron, H. Korzen, H. K. Müller (éds), Grammatica, hommages à Michael Herslund, Berne, Peter Lang, pp. 343-359.

Neveu, Franck, 2004, Dictionnaire des sciences du langage, Paris, Armand Colin.

Rabatel, Alain, 2003, «Les verbes de perception en contexte d'effacement énonciatif : du point de vue représenté aux discours représentés », Travaux de linguistique, ${ }^{\circ}$ 46, pp.49-88.

Rabatel, Alain, 2004/4, « La déliaison des énonciateurs et des locuteurs dans la presse satirique », Langage et société, $\mathrm{n}^{\circ} 110$, pp. 7-23.

Rabatel, Alain, 2005/4, «Le point de vue, une catégorie transversale », Le français aujourd'hui, $\mathrm{n}^{\circ}$ 151, pp. 57-68.

Rabatel, Alain, 2007, « Les enjeux des postures énonciatives et de leur utilisation en didactique », Éducation et didactique, vol. 1, nº 2, pp. 89-116.

Rabatel, Alain, 2008a, Homo narrans. Pour une analyse énonciative et interactionnelle du récit, t. 1, t. 2, Limoges, Lambert-Lucas.

Rabatel Alain, 2008b, « Retour sur les relations entre locuteurs et énonciateurs. Des voix et des points de vue », in M. Colas-Blaise, M. Kara, L. Perrin, Des voix et des points de vue, Luxembourg, Celted, Université de Metz, pp. 357-373.

Rabatel, Alain, 2012a, « Positions, positionnements et postures de l'énonciateur », Travaux neuchâtelois de linguistique, $\mathrm{n}^{\circ}$ 56, pp. 23-42.

Rabatel, Alain, 2012b, « Les relations Locuteur/Énonciateur au prisme de la notion de voix », Arts et savoirs, $\mathrm{n}^{\circ} 2$.

Rabatel, Alain, 2013, « Écrire les émotions en mode empathique », Semen, nº 35.

Rabatel, Alain, 2014, « Empathie, points de vue, méta-représentation et dimension cognitive du dialogisme ", ÉLA, Études de linguistique appliquée, n ${ }^{\circ}$ 173, pp. 27-45.

Rabatel, Alain, Lepoire, Soveig, 2005, « Le dialogisme des discours représentés et des points de vue dans les explications, entre concordance et discordance ", Cahiers de praxématique, $\mathrm{n}^{\circ} 45, \mathrm{pp}$. 51-76.

Rastier, François, 2002, Une introduction aux sciences de la culture, Paris, PUF.

Ricoeur, Paul, 1983-1985, Temps et récit, t. 1, t. 2 et t. 3, Paris, Seuil.

Tesnière, Lucien, 1959, Éléments de syntaxe structurale, Paris, Éditions Klincksieck.

Zilberberg, Claude, 2011, Des formes de vie aux valeurs, Paris, P.U.F.

Zilberberg, Claude, 2012, La structure tensive suivi de Note sur la structure des paradigmes et de Sur la dualité de la poétique, Liège, Presses universitaires de Liège. 


\section{NOTES}

1. Dans le sillage d'Oswald Ducrot, Alain Rabatel (p. ex. 2004) considère le locuteur (L) comme l'instance qui profère un énoncé dans ses dimensions phonétiques et phatiques ou scripturales, et l'énonciateur (E) comme l'origine d'un point de vue (PDV), défini comme un contenu propositionnel en relation avec des choix de référenciation pris en charge par l'énonciateur. Il distingue ainsi deux instances d'énonciation, le « locuteur, d'un côté, défini par la profération ou scription d'une actualisation langagière de longueur variable et l'énonciateur, de l'autre, défini comme un sujet modal à partir duquel la construction des productions langagières est envisagée, dans une optique radicale de l'énonciation, co-extensive à la langue en discours [...]» (2012b).

2. Au sujet de la narrativité « inchoative», voir Raphaël Baroni (2010), qui cite Ricoeur : s'il y a «promotion de sens, avancée de sens, de l'inchoatif au pleinement déterminé » (1985: 317-318), il faut prévoir la possibilité d'un « arrière-plan » qui « est fait de l'“imbrication vivante" de toutes les histoires vécues les unes dans les autres. Il faut donc que les histoires racontées "émergent" (Auftauchen) de cet arrière-plan. [...] Raconter, suivre, comprendre des histoires n'est que la “continuation" de ces histoires non dites» $(1983: 115)$. Nous dirons que la structure actantielle sous-tendant la perception est ancrée dans l'expérience sensible, avant toute narrativisation réalisée.

3. Pour le terme «attitude», on peut également se reporter à Ducrot (1984: 204) : «J'appelle "énonciateurs" ces êtres qui sont censés s'exprimer à travers l'énonciation, sans que pour autant on leur attribue des mots précis ; s'ils "parlent", c'est seulement en ce sens que l'énonciation est vue comme exprimant leur point de vue, leur position, leur attitude, mais non pas, au sens matériel, leurs paroles ».

4. Voir Rabatel (2005) pour la définition du PDV : «Le PDV correspond donc à ce qui, dans la référenciation linguistique des objets (du discours) révèle, d'un point de vue cognitif et axiologique, une source énonciative particulière et indique, explicitement ou implicitement, ses représentations, et, éventuellement, ses jugements sur les référents ». Alors que la perception n'est pas envisagée, ici, dans un cadre dialogique, la posture énonciative réclame l'interaction entre énonciateurs et l'entrée en contact de leurs PDV. La perception correspond à la première phase d'une actualisation.

5. Nous avons affaire à un "jeu de postures énonciatives ", ainsi que le notent Alice Pitoizet, Francis Grossmann et Agnès Tutin dans ce même numéro.

6. Nous renvoyons à Un balcon en forêt, 1958, Paris, José Corti.

7. Je remercie vivement Alain Rabatel d'avoir lu et commenté ce texte. Si la co-énonciation suppose l'ouverture à d'autres sujets percevants, celle-ci est facilitée par le fait qu'elle engage une interprétation «normale » (ni excessive ou partiale, ni incomplète ou partielle). D'où aussi l'idée d'une co-énonciation auto-dialogique (entre le soi percevant et le soi analysant).

8. Voir Rabatel (2012a : 27) au sujet de la prise en charge et de la prise en compte : dans le cas du DD et du DI, «E1 ne prend en charge que le fait de rapporter que Pierre lui a dit quelque chose hier, mais se borne à prendre en compte (Roulet 1981 : 19) l'engagement de venir ».

9. Le savoir établi peut faciliter la co-énonciation auto-dialogique.

10. Voir Zilberberg (2011) : le mode d'efficience est dirigé par le tempo, la vitesse commandant au survenir et la lenteur au parvenir. Si le mode d'existence correspond à l'état d'activation ou de passivation du sujet, sur la base de l'articulation visée vs saisie, le mode de jonction subsume au plan de l'expression l'opposition entre l'implication (dirigée par le parvenir) et la concession (dirigée par le survenir).

11. Dans le cadre du colloque qui lui a été consacré, Alain Rabatel s'interroge sur la possibilité de la concordance concordante dans le désaccord et le conflit. Il propose de mettre la concordance concordante en relation avec un dissensus proposé (co-énonciation d'un PDV opposé à un PDV 
autre) et la discordance discordante en relation avec un nouvel accord, après rejet du désaccord (énonciation d'un PDV alternatif qui constitue la base d'un accord potentiel).

12. D'où deux rythmiques différentes.

13. On trouve un écho à cette question dans ce volume, dans l'analyse des détournements proposée par Émilie Devriendt.

14. Dans sa communication au colloque de 2018, Hugues Constantin de Chanay parle de postures «performées ». L'idée de la performance mérite d'être développée et mise en relation avec celle de la performativité. Nous privilégions une approche énonciative interactionnelle dynamiciste : l'accord ou le désaccord ne constituent pas un préalable, mais les négociations entre énonciateurs les font exister.

15. Alain Rabatel propose un continuum (accord, prise en compte, désaccord). La consonance est un accord implicite, la dissonance un désaccord implicite.

16. Je développe ici une suggestion d'Alain Rabatel (colloque).

17. On pourrait élargir la notion de co-énonciation en considérant que même le désaccord réclame un espace énonciatif partagé.

18. Au sujet du désaccord limitant la co-construction d'un PDV commun en faisant surgir un PDV « alternatif » cf. Alain Rabatel (2012a : 38).

19. C. Jullien (2012: 7) : «[...] l'écart relève d'une logique d'immanence, de part en part, et n'en déborde d'aucun côté, sans donc donner à postuler. De plus, ne relevant pas de l'établissement d'une distinction, mais procédant d'une distance, il s'agit là d'un concept qui, faisant entendre le mouvement de séparation dont il est né, n'est pas tant analytique que dynamique, procédant d'un auto-déploiement. Le propre de l'écart - et c'est là pour moi l'essentiel - c'est qu'il n'est, par suite, pas proprement aspectuel ou descriptif, comme l'est la différence, mais productif - et ce dans la mesure même où il met en tension ce qu'il a séparé. Mettre en tension : c'est à quoi l'écart doit d'opérer ".

20. Voir Jullien (ibid.: 6) : «[...] l'établissement des différences suppose que je m'arroge une position de surplomb, ou du moins d'extériorité, à partir de laquelle c'est moi qui range entre le même et l'autre, l'identique et le différent, et qui compare. Or quelle est cette extériorité - extraterritorialité culturelle - dont je bénéficierais (où la situer ?) d'où je puisse ainsi tout mettre à plat, devant moi, et comparer ».

21. Voir Alain Rabatel (2013) : «Un centre d'empathie correspond à un énonciateur interne (codé e2), distinct du locuteur/énonciateur premier (L1/E1). [...] Enfin, par modalités d'empathie, j'entends les différentes collocations de marques indiquant comment E1 imagine ce que e2 pourrait dire, penser, percevoir, ressentir, faire». Il ajoute (2014:29-30) que «l'empathie est donc une aptitude à se mettre à la place des autres, sans fusion ni identification, tandis que la sympathie consiste en une identification aux autres avec partage de leurs émotions ». Enfin, il parle de «consonance émotionnelle ». Il semblerait que, dans le cas de la « diathèse réciproque fusionnée ", il s'agisse de sympathie, alors que l'empathie caractérise la diathèse récessive, que nous rapprochons de la co-énonciation.

22. Ce mouvement empathique peut constituer un trait stylistique du roman Un balcon en forêt (si l'on inclut dans la notion de style une composante expérientielle et interactionnelle) (ColasBlaise 2012)).

23. L'événement perceptif a son équivalent au niveau de l'écriture : l'événement énonciatif en est le pendant. Plus largement, l'entrecroisement des versions consensuelle et dissensuelle de la coénonciation témoigne d'un style d'écriture qui est redondant avec les glissements qui s'opèrent au plan du contenu. Le style d'écriture comprend deux autres composantes: un style praxique (une manière de se situer par rapport à d'autres discours, par exemple des énoncés doxiques) et un style expérientiel (une manière de prendre position devant ou dans le monde) (Colas-Blaise 2012). C'est ce que j'essayerai de montrer dans la troisième partie. 
24. Voir Rabatel (2003) : «Sur le plan sémantique, la parenté des verba sentiendi et dicendi fait du PDV un mixte de pensées, de perceptions et de paroles qui le rapproche du DIL (en contexte narratif à la troisième personne) ou du DDL (en contexte narratif à la première personne), jouant un rôle décisif dans l'expression d'une sorte de pensée pré-réflexive. Ces phénomènes renvoient à un continuum, des formes réflexives aux formes pré-réflexives, et plaident pour une redéfinition du DR comme discours représenté plutôt que rapporté »

25. En ce qui concerne la perception et la genèse du sens, l'on se reportera également au groupe $\mu(2015)$.

26. Ailleurs, le présent générique peut relever de la sur-énonciation. Dans un Balcon en forêt, le régime de la co-énonciation est à tel point prégnant (par opposition aux régimes de la sur- et de la sous-énonciation) que l'interprétation du présent générique elle-même en est affectée. À cela s'ajoute que le présent "omnitemporel», qui se distingue du présent chargé de la valeur d'actualité, amorce une remontée vers le temps pré-réflexif des premières chronothèses (cf. Combe 1991 : 163).

27. Sans doute le déterminant démonstratif « cette » peut-il se charger également d'une valeur cataphorique (relative complétant un $\mathrm{SN}$ démonstratif).

28. Significativement, le verbe conjugué est relégué dans une proposition relative adjective au second plan, l'action étant non bornée.

29. Prenant ses distances par rapport à l'analyse du démonstratif par les praxématiciens, Michèle Monte (2011 : 126) parle d'un « espace intersubjectif indifférencié ».

30. Le « proto-embrayage » selon Denis Bertrand (2005) consiste à « donner langue et sens à une instance antérieure, plus originaire, plus "génitive", se tenant au plus près de l'engendrement, au plus près de la présence corporelle à partir de l'impression sensible ».

31. Le positionnement plonge dans le monde à peine sémiotisé ; le proto-embrayage correspond à l'être-temps de l'immersion. La prise de position est opérée par la perception. Quant à la posture, il ne s'agit pas seulement de prendre position dans le monde, perçu, aspectualisé, temporalisé et représenté, mais encore d'être là, dans le monde et dans le temps.

\section{RÉSUMÉS}

Cet article, qui s'appuie sur Un balcon en forêt de Julien Gracq, se propose de réinterroger la notion de posture énonciative développée par Alain Rabatel dans sa théorie du point de vue. L'attention est focalisée sur la posture de la co-énonciation, qui est abordée sous différents angles : du point de vue de la diathèse en particulier récessive, qui est mise en relation avec l'opération de l'embrayage et une visée centripète; du point de vue des régimes tonique et atone de la coénonciation consensuelle et dissensuelle; enfin, du point de vue d'une proto-posture énonciative sous-tendant la posture de la co-énonciation, qui permet de rendre compte de la gestation du sens et des couches sensibles du pathique.

This paper, which analyses extracts of Le balcon en forêt by Julien Gracq, aims to question once more the notion of enunciative posture that has been examined by Alain Rabatel in his point of view theory. Attention is focused on the posture of co-enunciation, which is approached from three different viewing angles: first, we reflect upon the notion of "recessive diathesis", in relation to a centripetal force; second, we distinguish between tonic and low regimes of 
consensual and dissensual co-enunciation; third, we want to show that the posture of coenunciation is based on a sensitive proto-posture, which underlies it.

\section{INDEX}

Keywords : co-enunciation, diathesis, regimes of consensual and dissensual co-enunciation, enunciative proto-posture

Mots-clés : co-énonciation, diathèse, régimes de la co-énonciation consensuelle et dissensuelle, proto-posture énonciative

\section{AUTEUR}

MARION COLAS-BLAISE

Université du Luxembourg 